\title{
DEVELOPMENT OF A THEORETICAL MODEL FOR A SOLVENT-TYPE PREPREG MANUFACTURING PROCESS
}

\author{
Jiaching Liu, * Diing-Guey Hwang, John-Chang Chen \\ Materials R\&D Center, Chung-Shan Institute of Science and Technology, PO Box 90008-8-2, Lung-Tan, Taiwan 32500
}

$\&$

Wen-Jei Yang

Department of Mechanical Engineering and Applied Mechanics, University of Michigan, Ann Arbor, Michigan 48109-2125, USA

(Received 6 January 1993; revised version received 31 August 1993; accepted 1 October 1993)

\begin{abstract}
This paper presents a theoretical study of the impregnation of continuous fiber or cloth with thermosetting resin matrix materials. A model is developed to describe the resin, the volatile content and the coated resin thickness as functions of impregnation velocity for a solvent-type, vertical impregnating process. A power law is employed to account for the effect of shear rate on the resin velocity in the model. Results are obtained methodically through the separation of variables. A dimensionless number, called the impregnation number, is derived which expresses the relative importance among the viscous, inertial and gravity forces in the impregnation process. It is found that the dimensionless number is important in characterizing the maximum possible resin content for a given condition, and that an increase in the resin viscosity is much more effective than an increase in the impregnation velocity in achieving higher resin content. The volatile content is controlled by a combination of the impregnation velocity and the oven length. The predicted volatile contents agree well with the experimental results for a $177^{\circ} \mathrm{C}$ prepreg. The model is valid for predicting volatile content in the practical impregnation process.
\end{abstract}

Keywords: impregnation velocity, solvent-type prepreg, resin content, volatile content

\section{NOTATION}

$D_{\mathrm{AB}} \quad$ Mass diffusivity $\left(\mathrm{m}^{2} / \mathrm{s}\right)$

$g \quad$ Acceleration of gravity $\left(\mathrm{m} / \mathrm{s}^{2}\right)$

$k_{\mathrm{L} \text {.ave }}$ Average mass transfer coefficient $(\mathrm{m} / \mathrm{s})$

* To whom correspondence should be addressed. $k_{\text {If }} \quad$ Mass transfer coefficient of film theory $(\mathrm{m} / \mathrm{s})$

$L \quad$ Length of the oven or drying path (m)

$n \quad$ Flow index (dimensionless)

$n_{\mathrm{A}} \quad$ Mass flux of volatile $\left(\mathrm{kg} / \mathrm{s} \mathrm{m}^{2}\right)$

$N_{\text {Im }} \quad$ Impregnation number (dimensionless)

$T_{\mathrm{g}} \quad$ Thickness of the reinforcement (m)

$U_{\mathrm{y}} \quad$ Velocity in $y$ direction $(\mathrm{m} / \mathrm{s})$

$\vec{U} \quad$ Impregnation velocity $(\mathrm{m} / \mathrm{s})$

$X_{\mathrm{A}} \quad$ Normalized volatile content

$\overline{X_{\mathrm{A}}} \quad$ Normalized volatile content averaged in $y$-direction

$y \quad$ Cartesian coordinate measured from oven inlet $(\mathrm{m})$

$z \quad$ Cartesian coordinate measured from the surface of the resin layer $(\mathrm{m})$

$\dot{\gamma} \quad$ Shear rate $\left(\mathrm{s}^{-1}\right)$

$\dot{\gamma}_{0} \quad$ Reference shear rate state (taken as $1 \mathrm{~s}^{-1}$ )

$\delta \quad$ Thickness of the resin layer $(\mathrm{m})$

$\eta_{0} \quad$ Viscosity of resin at reference state $\left(\mathrm{Ns} / \mathrm{m}^{2}\right)$

$\mu \quad$ Apparent viscosity $\left(\mathrm{Ns} / \mathrm{m}^{2}\right)$

$\rho_{\mathrm{A}} \quad$ Density of the volatile $\left(\mathrm{kg} / \mathrm{m}^{3}\right)$

$\rho_{\mathrm{g}} \quad$ Density of the reinforcement $\left(\mathrm{kg} / \mathrm{m}^{3}\right)$

$\rho_{\mathrm{R}} \quad$ Density of the resin $\left(\mathrm{kg} / \mathrm{m}^{3}\right)$

$\omega_{\mathrm{A}} \quad$ Weight fraction of volatile (volatile content)

$\omega_{\mathrm{Ai}} \quad$ Weight fraction of volatile (volatile content) at interface between air and prepreg

$\omega_{\mathrm{AO}}$ Weight fraction of volatile (volatile content) entering heating oven

$\omega_{\mathrm{R}} \quad$ Weight fraction of resin (resin content) of prepreg

$\omega_{\mathrm{S}} \quad$ Weight fraction of resin (solid content) of varnish in dipping pan

Superscript

* Dimensionless quantities 


\section{INTRODUCTION}

In manufacturing fiber-reinforced-plastic composite components, the use of prepregs reduces the work involved and provides stronger, stiffer and more reliable parts than equivalent components produced by wet lay-up processes. But these advantages will only be realized when prepregs with consistent properties are used. Prepregs consist of a layer of fiber or cloth impregnated with a partial mixture of monomers or oligomers, either in a solvent (solventtype prepreg) or as a solvent-free medium (hot-melt type). In the case of a solvent-type prepreg, the impregnated reinforcement (prepreg) usually passes through a heating oven to decrease the solvent content. The mixture will continue to react and lose solvent to a degree determined by the storage and handling environments. Most of the available literature and technical reports ${ }^{1-3}$ dealing with prepregs emphasize either the formulation and quality assurance of incoming resins or various applications of prepregs. Those that discuss prepreg characterization and processing will be reviewed in the following paragraphs.

In the preparation of composites, two concepts (tack and drape) have been used to characterize the suitability of prepregs. Sanjana ${ }^{4}$ identified prepreg tack as the critical property after studying the aging of prepregs under various conditions. Loss of tack can be tolerated in the making of flat laminates but it is a problem in the manufacturing of parts with complex contours. It is suggested ${ }^{5}$ that, to a certain degree, tack can be adjusted by controlling the volatile content of a prepreg. Cole et al. ${ }^{\circ}$ discovered a significant loss of tack when the volatile content in a Narmco 5208 prepreg decreases from 1.5 to $0.8 \%$. A similar correlation between tack and volatile content was observed in American Cynamid BP-927 prepreg. ${ }^{6}$ Though a higher volatile content can increase the tackiness, it also affects the viscosity profile during curing process and produces porosity in the laminates. By means of differential scanning calorimetry (DSC), Yue $^{7}$ studied the effects of volatile content of prepregs and concluded that prepregs which have a lower volatile content would have a higher compressibility and ability to flow during processing. Day and Shepard $^{5}$ examined the advancement of epoxy resin by monitoring its viscosity profile through dielectric analysis. An initial difference was observed in the viscosity profiles which could have been due to the difference in volatile content. In addition to volatile content, an inhomogeneity in the resin content of prepregs could also be a problem in both the processing and performance of the final parts. A composite component may have different fiber/resin ratios for different regions as a result of this inhomogeneity of resin content. A difference in resin content also makes the necessary characterizations of prepreg more difficult. Unfortunately, differences of $5-10 \%$ in resin content were encountered in some of the commercial prepregs.

Although the resin and volatile contents are very important in determining the quality of prepregs from one batch to another, very little has been said regarding the factors that are crucial in controlling quality during the impregnation process. The theory of deposition of a viscous liquid onto a moving substrate was first investigated in detail by Deryagin $e t$ $a l^{8}$ It was shown that, depending upon the influence of capillary effect, the maximum possible thickness of a coating is proportional to a one-half or two-thirds power of the impregnation velocity. Levy et al." examined the combined effect of freeze coating, and viscous coating, and concluded that at the higher speeds and shallower baths, viscous coating is the predominating factor, obtaining satisfactory agreement of their theory and experiments. Rezaian and Poulikakos ${ }^{16}$ studied the heat and fluid flow processes of a moving isothermal surface by similarity analysis In addition to the Stefan number, the velocity ratio between the liquid depositing and the moving surface was found to be an important parameter in determining the coating thickness. Using a perturbation method, Cheung" studied a case involving a non-isothermal moving plate and showed that the freeze-coat would only grow within a limited distance from the inlet of liquid bath. Owing to the assumptions made in these studies, the results are more readily applied to hot-melt type impregnation process than to solvent-type process.

Despite the importance of solvent-type prepregs in composite manufacturing, several questions related to the mass transfer and fluid flow during the impregnation process remain unanswered. The objective of the present work was to model a vertical impregnation process and to develop a relationship between the impregnation velocity, resin content and volatile content. The system will be modeled as a viscous coating process with coordinate system held stationary with respect to the moving reinforcement. The resin is considered a non-Newtonian fluid and its viscosity is represented through the power law. The results improve understanding of the fundamental aspects of the impregnation process, and assist in maintaining the consistent quality of prepregs.

\section{FORMULATION}

The general layout for a vertical solvent-type impregnator is illustrated in Fig. 1, while the system to be analyzed is schematically defined in Fig. 2 . The continuous reinforcement leaves the dipping pan and enters into a heating oven with a constant velocity $\bar{U}$ and a volatile content of $\omega_{\mathrm{AO}}$ determined by the solid 


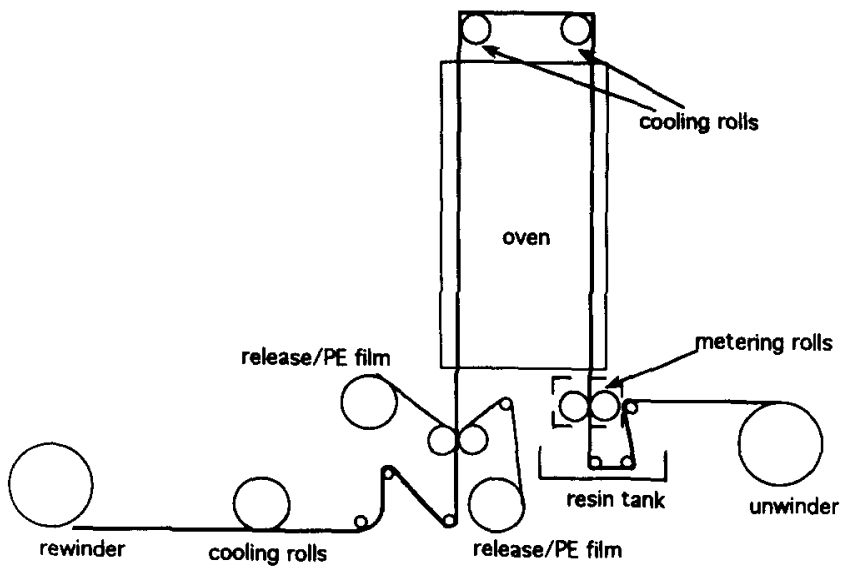

Fig. 1. A general layout of an impregnator.

content of the coated resin. The temperature inside the oven is maintained constant. The concentration of the volatile on the free surface (represent by the plane of $z=0$ ) is in equilibrium with the volatile concentration in heating air and is constant at $\omega_{\mathrm{Ai}}$. There is no velocity gradient on the free surface of the coated resin and a no-slip boundary condition at the interface between the resin and reinforcement. The

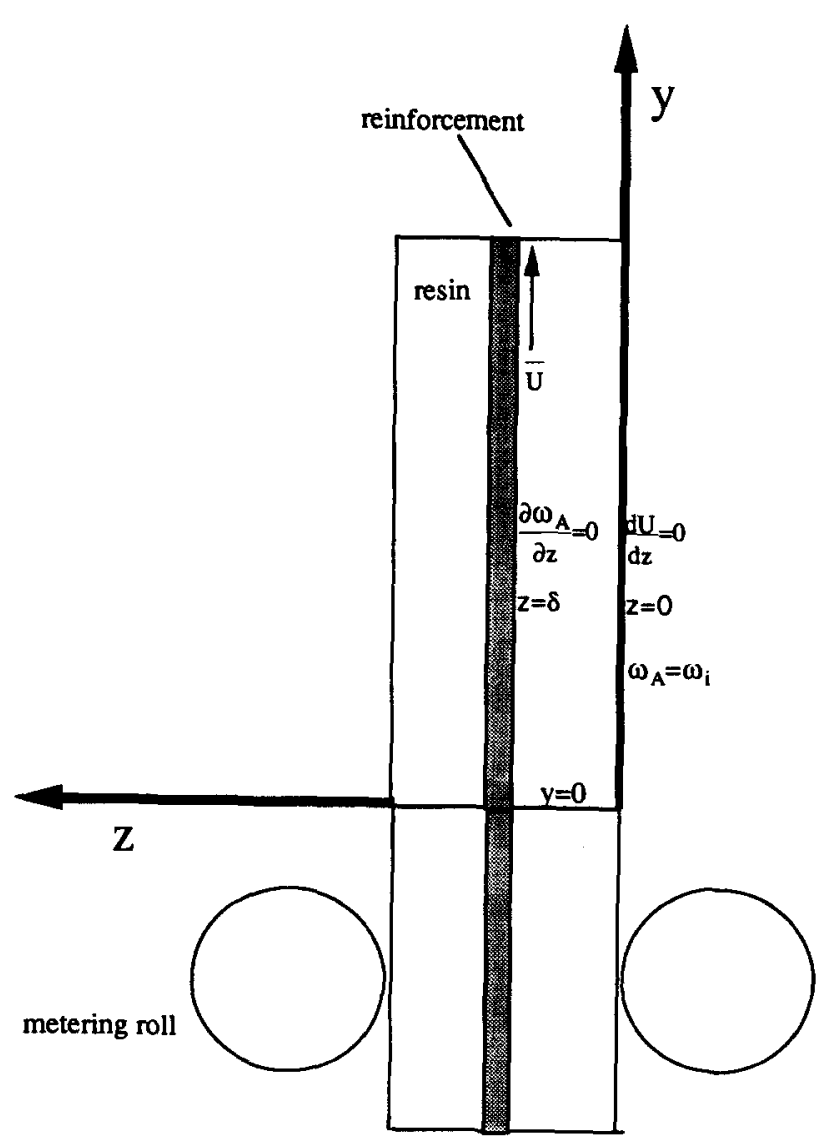

Fig. 2. A coordinate system of prepreg leaving dipping pan and entering heating oven. following assumptions have been made to simplify the problem: (1) the process is at steady state; (2) the temperature of the prepreg is lumped and is equal to the oven temperature; (3) the resin does not penetrate far into the reinforcements; (4) the viscosity is a function of temperature and shear rate only; (5) the volatile concentration in the heating air is negligibly small. Assumption (4) is adopted with the understanding that the present study is intended to determine the phenomenon in the process.

The equation of motion gives

$$
\frac{\mathrm{d}}{\mathrm{d} z}\left(\mu \frac{\mathrm{d} U_{y}}{\mathrm{~d} z}\right)-\rho_{\mathrm{R}} g=0
$$

where the viscosity of a B-stage resin, $\mu$, is assumed to be expressed through power law

$$
\mu=\eta_{0} \frac{\dot{\gamma}^{n-1}}{\dot{\gamma}_{0}} \text { or } \mu=\eta_{0}\left(\frac{\mathrm{d} U_{\mathrm{y}}}{\mathrm{d} z}\right)^{n-1}
$$

when taking $\dot{\gamma}_{0}=1 \cdot 0$. The mass balance of the volatile yields

$$
\frac{U_{\mathrm{y}} \partial \omega_{\mathrm{A}}}{D_{\mathrm{AB}} \partial y}=\frac{\partial^{2} \omega_{\mathrm{A}}}{\partial z^{2}}
$$

Equations (1) and (2) are subject to the following boundary conditions:

- at the free surface of the coated resin layer $(z=0)$ :

$$
\begin{aligned}
\frac{d U_{\mathrm{y}}}{\mathrm{d} z} & =0 \\
\omega_{\mathrm{A}} & =\omega_{\mathrm{Ai}}
\end{aligned}
$$

- at the interface between resin and reinforcement $(z=\delta)$ :

$$
\begin{aligned}
U_{\mathrm{y}} & =\bar{U} \\
\frac{\partial \omega_{\mathrm{A}}}{\partial z} & =0
\end{aligned}
$$

- at the entrance of the heating oven $(y=0)$ :

$$
\omega_{\mathrm{A}}=\omega_{\mathrm{AO}}
$$

Equation (3) neglects both the shear force on air and the surface tension induced flow on the free surface. Since surface tension induced flow is usually caused by either the surface tension gradient or the curvature on the free surface, the assumptions of isothermal condition and zero curvature have precluded it from happening. Equation (6) implies that no volatile penetrates the reinforcement during impregnation process. This is a reasonable approximation, as no squeezing pressure is applied and mass transfer is dominated by diffusion process. 


\section{SOLUTION}

\section{Resin content}

Equation (1) is solved subject to boundary conditions eqns (3) and (5), yielding

$$
U_{\mathrm{y}}=\bar{U}-\frac{n}{n+1}\left(\frac{\rho_{\mathrm{R}} g}{\eta_{0}}\right)^{1 / n} \delta^{(n+1) / n}\left[1-\left(\frac{z}{\delta}\right)^{(n+1) / n}\right]
$$

Equation (8) can be rewritten in the dimensionless form as

$$
U_{\mathrm{y}}^{*}=1-\frac{n}{n+1}\left(\frac{\rho_{\mathrm{R}} g \delta^{n+1}}{\eta_{0} \bar{U}^{n}}\right)^{1 / n}\left[1-z^{*(n+1) / n}\right]
$$

where

$$
U_{\mathrm{y}}^{*}=\frac{U_{\mathrm{y}}}{\bar{U}} \quad \text { and } \quad z^{*}=\frac{z}{\delta}
$$

The absence of dripping and backflow of coated resin requires $U_{\mathrm{y}}^{*} \geq 0$ at $z^{*}=0$. Substituting this condition into eqn (9), one obtains

$$
N_{\mathrm{Im}} \leq\left(\frac{n+1}{n}\right)^{n}
$$

Here, the impregnation number is defined as

$$
N_{\mathrm{Im}}=\frac{\rho_{R} g \delta^{n+1}}{\eta_{0} \bar{U}^{n}}
$$

For a Newtonian fluid, eqn (10) reduces to $n=1$ and eqn (10) reduces to

$$
N_{\text {Im }} \leq 2
$$

The maximum possible thickness of a coated layer for a given velocity can be found by letting $U_{\mathrm{y}}=0$ at $z=0$ in eqn (8). This yields

$$
\delta_{\max }=\left(\frac{n+1}{n}\right)^{n /(n+1))}(\bar{U})^{n /(n+1)}\left(\frac{\eta_{0}}{\rho_{\mathrm{R}} g}\right)^{1 /(n+1)}
$$

The resin content of a prepreg is defined as

$$
\omega_{\mathrm{R}}=\frac{\rho_{\mathrm{R}} \delta_{\max }}{\rho_{\mathrm{R}} \delta_{\text {max }}+\rho_{\mathrm{g}} \frac{T_{\mathrm{g}}}{2}}
$$

where

$$
\rho_{\mathrm{R}}=\omega_{\mathrm{s}} \rho_{\mathrm{s}}+\omega_{\mathrm{AO}} \rho_{\mathrm{A}}
$$

A relationship between resin content and impregnation velocity is derived by combining eqns (12) and (13). This yields

$$
\begin{aligned}
\bar{U}= & \left(\frac{n}{n+1}\right)\left[\frac{\left(\omega_{\mathrm{s}} \rho_{\mathrm{s}}+\omega_{\mathrm{AO}} \rho_{\mathrm{A}}\right) g}{\eta_{0}}\right]^{1 / n} \\
& \times\left[\frac{\omega_{\mathrm{R}}}{\omega_{\mathrm{s}}\left(1-\omega_{\mathrm{R}}\right)}\right]^{(n+1) / n}\left(\frac{\rho_{\mathrm{g}}}{\omega_{\mathrm{s}} \rho_{\mathrm{s}}+\omega_{\mathrm{AO}} \rho_{\mathrm{A}}}\right)^{(n+1) / n} \\
& \times\left(\frac{T_{\mathrm{g}}}{2}\right)^{(n+1) / n}
\end{aligned}
$$

\section{Volatile content}

Without dripping or backflow, a normal impregnation process, the velocity $U_{\mathrm{y}}$, may be treated constant at $\bar{U}$. Equation (2) then becomes

$$
\frac{\bar{U} \partial \omega_{\mathrm{A}}}{\mathrm{D}_{\mathrm{AB}} \partial y}=\frac{\partial^{2} \omega_{\mathrm{A}}}{\partial z^{2}}
$$

subject to the boundary conditions, eqns (4), (6) and (7).

The nonhomogeneity in eqn (7) can be eliminated through the definition of a new variable,

$$
X_{\mathrm{A}}=\frac{\omega_{\mathrm{A}}-\omega_{\mathrm{Ai}}}{\omega_{\mathrm{AO}}-\omega_{\mathrm{Ai}}}
$$

The governing eqn (2) is then reduced to

$$
\frac{\bar{U} \partial X_{\mathrm{A}}}{D_{\mathrm{AB}} \partial y}=\frac{\partial^{2} X_{\mathrm{A}}}{\partial z^{2}}
$$

subject to the boundary conditions

$$
\begin{gathered}
X_{\mathrm{A}}=1, \quad y=0 \\
X_{\mathrm{A}}=0, \quad z=0 \\
\frac{\partial X_{\mathrm{A}}}{\partial z}=0, \quad z=\delta
\end{gathered}
$$

The solution is obtained as

$$
X_{\mathrm{A}}(y, z)=\sum_{n=1}^{\infty} b_{n} \exp \left(\frac{-\alpha_{n}^{2} D_{\mathrm{AB}} y}{\bar{U}}\right) \sin \alpha_{n} Z
$$

where

$$
b_{n}=\frac{4}{(2 n-1) \pi}, \quad \alpha_{n}=\frac{(2 n-1) \pi}{2 \delta}
$$

In order to develop a relationship between volatile content at the exit of the oven and the impregnation velocity, the concentration is integrated in the $z$-direction yielding

$$
\begin{aligned}
\overline{X_{\mathrm{A}}}(y) & =\frac{1}{\delta} \int_{0}^{\delta} \sum_{1}^{\infty} b_{n} \exp \left(\frac{-\alpha_{n}^{2} D_{\mathrm{AB}} y}{\bar{U}}\right) \sin \alpha_{z} z \mathrm{~d} z \\
& =\frac{8}{\pi^{2}} \sum_{1}^{\infty} \frac{1}{(2 n-1)^{2}} \exp \left(\frac{-\alpha_{n}^{2} D_{\mathrm{AB}} y}{\bar{U}}\right)
\end{aligned}
$$

\section{Mass transfer coefficient}

The mass flux of the volatile at $z=0$ is

$$
\begin{aligned}
n_{\mathrm{A}} & =\rho_{\mathrm{R}} D_{\mathrm{AB}}\left(\frac{\partial \omega_{\mathrm{A}}}{\partial z}\right)_{z=0} \\
& =\frac{2 \rho_{\mathrm{R}} D_{\mathrm{AB}}}{\delta}\left(\omega_{\mathrm{AO}}-\omega_{\mathrm{Ai}}\right) \sum_{1}^{\infty} \exp \left(\frac{-\alpha_{n}^{2} D_{\mathrm{AB}} y}{\tilde{U}}\right)
\end{aligned}
$$

Hence, the average mass flux for an oven length of $L$ 
is found to be

$$
\begin{aligned}
n_{\mathrm{A}, \text { ave }}= & \frac{\int_{0}^{L} n_{\mathrm{A}} \mathrm{d} y}{\int_{0}^{L} \mathrm{~d} y}=\frac{2 \bar{U} \delta \rho_{\mathrm{R}}}{L}\left(\omega_{\mathrm{AO}}-\omega_{\mathrm{Ai}}\right) \\
& \times \frac{4}{\pi^{2}} \sum_{n=1}^{\infty} \frac{1}{(2 n-1)^{2}}\left[1-\exp \left(\frac{-\alpha_{n}^{2} D_{\mathrm{AB}} L}{\bar{U}}\right)\right]
\end{aligned}
$$

Subsequently, one obtains the average mass transfer coefficient as

$$
k_{\mathrm{L}, \mathrm{av}}=\frac{2 \tilde{U} \delta}{L} \frac{4}{\pi^{2}} \sum_{n=1}^{\infty} \frac{1}{(2 n-1)^{2}}\left[1-\exp \left(\frac{-\alpha_{n}^{2} D_{\mathrm{AB}} L}{\bar{U}}\right)\right]
$$

or in the dimensionless form as

$$
\begin{aligned}
\frac{k_{\mathrm{L}, \mathrm{av}}}{k_{\mathrm{L}, \mathrm{f}}}= & \frac{k_{\mathrm{L}, \mathrm{av}}}{D_{\mathrm{AB}} / \delta}=\frac{2 \bar{U} \delta^{2}}{D_{\mathrm{AB}} L} \sum_{n=1}^{\infty} \\
& \times \frac{4}{\pi^{2}} \frac{1}{(2 n-1)^{2}}\left[1-\exp \left(\frac{-\alpha_{n}^{2} D_{\mathrm{AB}} L}{\bar{U}}\right)\right]
\end{aligned}
$$

\section{RESULTS AND DISCUSSION}

A dimensionless number, called the impregnation number, is derived which controls the success or failure of a viscous coating process. It signifies the ratio of the gravity force to the product of the viscous and inertial forces:

$$
N_{\mathrm{Im}}=\frac{\rho_{\mathrm{R}} g \delta^{n+1}}{\eta_{0} \bar{U}^{n}}=\frac{\text { gravity force }}{(\text { viscous force }) \times(\text { inertial force })}
$$

Equation (10) is the criterion for the occurrence of an impregnation process without dripping. It requires the product of viscous and inertial forces must be greater than the gravitational force by a factor of $((n+1) / n)^{n}$.

Since impregnation velocity and viscosity can be easily controlled during an impregnation process, it is of importance to know the influence of these factors on resin content and volatile content for prepregs. Equation (12) is graphically presented in Figs 3 and 4. Figure 3 is a plot of the maximum thickness versus the impregnation velocity with the viscosities and flow index as parameters. For a Newtonian fluid $(n=1)$, the coated thickness increase significantly with an increase in the impregnation velocity compared with that of a shear-thinning fluid $(n=1 / 2)$ with the same viscosity. Under the same viscosity, the coated thickness of a shear-thinning fluid approaches its asymptotic value at a much lower impregnation velocity than that of a Newtonian fluid due to a build up of the shearing force induced by the velocity gradient within the coated layer. In order to increase the coated thickness under such circumstance, it is more efficient to change the rheological properties than to increase the impregnation velocity, as shown

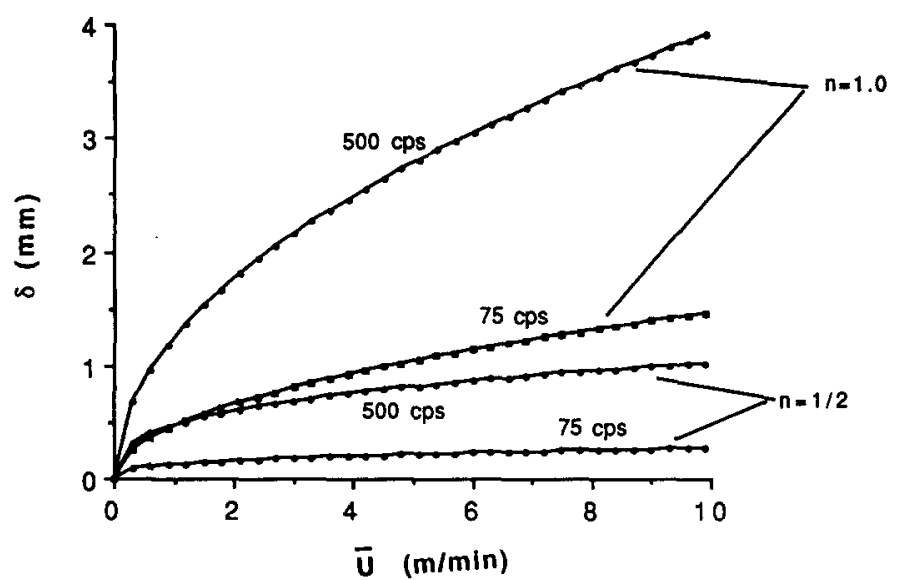

Fig. 3. Effect of impregnation velocity on the maximum possible thickness.

in Fig. 4. It is seen that the coated thickness increases almost linearly with an increase in viscosity for both Newtonian and shear-thinning materials.

Equation (14) is plotted in Fig. 5 for $n=1.0$ and $\eta_{0}=75 \mathrm{cps}$. It is seen that for $\bar{U}$ less than $0.5 \mathrm{~m} / \mathrm{min}$, an increase in solid content produces little effect on the resin content. As $\bar{U}$ is increased, the solid content plays an important role on resin content at a given impregnation velocity. In other words, the impregnation velocity may be adjusted to achieve a desired resin content for prepreg, depending on the solid content of the resin: Equation (14) is replotted in Fig. 6 to demonstrate the effect of shear-thinning on the resin content, for $\eta_{0}=75 \mathrm{cps}$ and a solid content of 0.9. Again, the shear-thinning fluid reaches an asymptotic value much faster than a Newtonian fluid at a higher impregnation velocity. It is seen in Figs 3 and 6 that, in order to obtain a higher resin content in a prepreg, a change in the rheological properties of a

$\overline{\mathrm{U}}=6.0 \mathrm{~m} / \mathrm{min}$

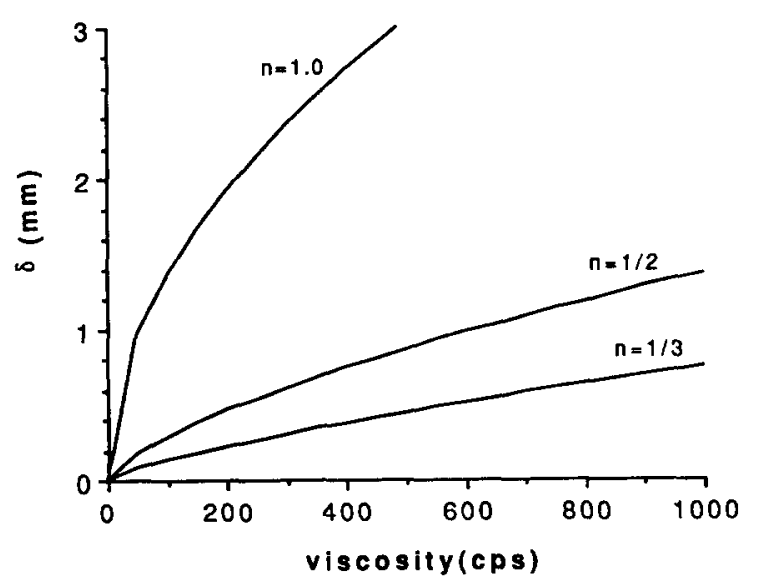

Fig. 4. Effect of viscosity and flow index on the maximum possible thickness. 


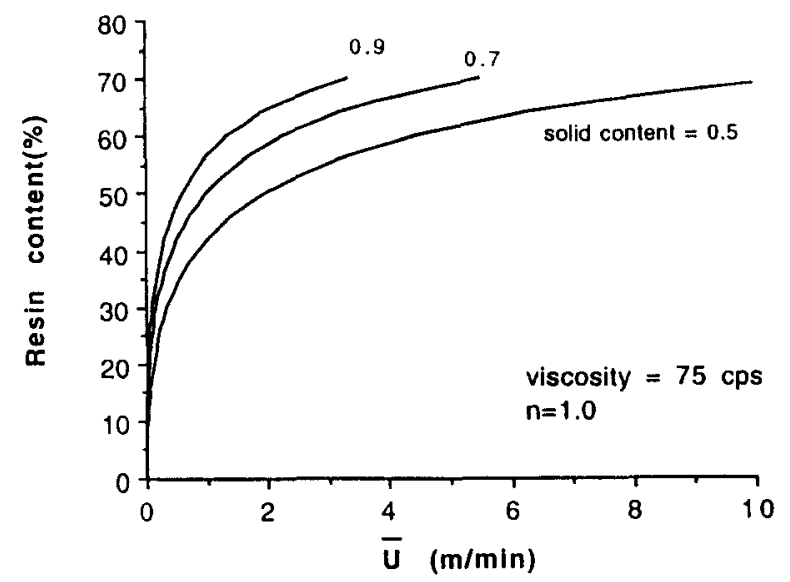

Fig. 5. Effect of impregnation velocity on resin content with various solid content.

resin, such as viscosity or flow index, is more effective than to increase the impregnation velocity.

Figure 7 is another graphical illustration of eqn (14). It depicts the operating condition necessary for an impregnation process to achieve $45 \%$ resin content of a prepreg for a shear-thinning resin. An increase in the viscosity results in a reduction in the impregnation velocity, beneficial in the removal of the volatile of a prepreg, as will be explained in the following paragraph. At a given viscosity, the impregnation velocity can be decreased to meet the B-stage conditioning simply by increasing the solid content of the resin.

It is known that volatiles can increase both the tackiness of prepreg and the potential sources of porosity of the final parts. One important parameter that determines the amount of volatile remaining after the impregnation process is the diffusion coefficients of volatiles in B-stage resins. The well known Wilke-Chang correlation is only valid for dilute solution, ${ }^{12}$ thus an estimated value of diffusivity will be used in computation. Since the diffusion

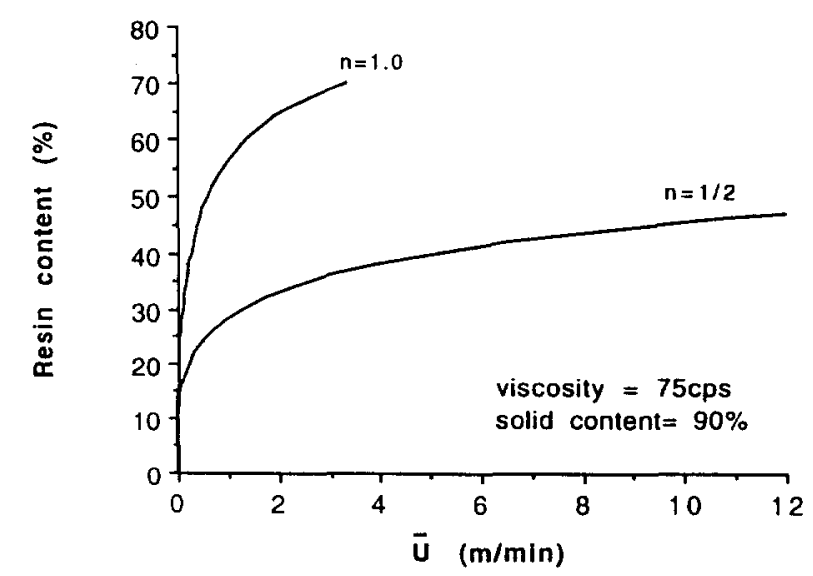

Fig. 6. Effect of impregnation velocity on resin content with various flow indices.

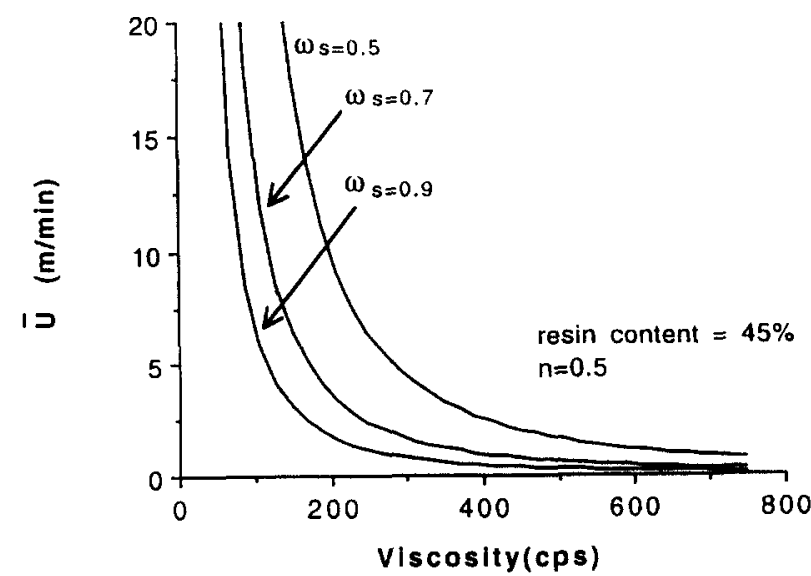

Fig. 7. Effect of viscosity on impregnation velocity to attain a resin content of $45 \%$ for a shear-thining resin.

coefficients are about $10^{-5} \mathrm{~cm}^{2} \mathrm{~s}$ for liquids and $10^{-8} \mathrm{~cm}^{2} / \mathrm{s}$ for glasses and solid polymers, ${ }^{13}$ those for volatiles in resin lie between these two limits. A value of $10^{-6} \mathrm{~cm}^{2} / \mathrm{s}$ is used to compute the volatile content, $\overline{X_{\mathrm{A}}}(y)$ in eqn (21).

Results are illustrated in Figs 8-10. Note that the ordinate is the normalized volatile content. One may use Fig. 8 determine the magnitudes of the impregnation velocity and heating length for a desired volatile content. For example, to reach a $50 \%$ $\left(X_{\mathrm{A}}=50 \%\right)$ normalized volatile content for an absolute volatile content of $5 \%\left(\omega_{\mathrm{A}}=5 \%\right)$ one may select either $U=4.8 \mathrm{~m} / \mathrm{min}$ and a heating distance of $4 \mathrm{~m}$, or $\bar{U}=2.4 \mathrm{~m} / \mathrm{min}$ and a heating length of $2 \mathrm{~m}$. For ovens with a fixed heating length, the impregnation velocity is a key factor in controlling the volatile content. Figure 9 is a plot of the normalized volatile content versus the impregnation velocity in an oven of $14 \mathrm{~m}$ length. It is seen that the volatile content is linearly dependent on $\bar{U}$ for $\bar{U}$ exceeding 2 . Theoretical prediction is compared with experimental

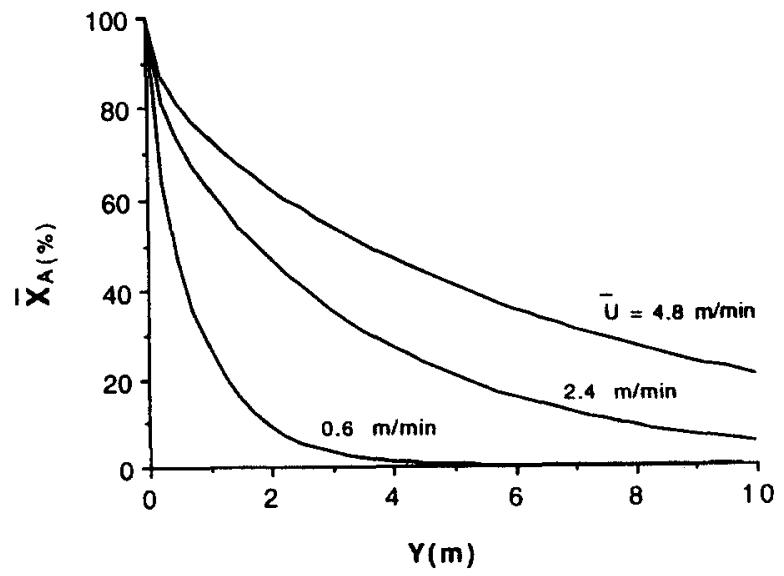

Fig. 8. Volatile content versus distance from the inlet of heating oven with $\omega_{R}=0.45$. 


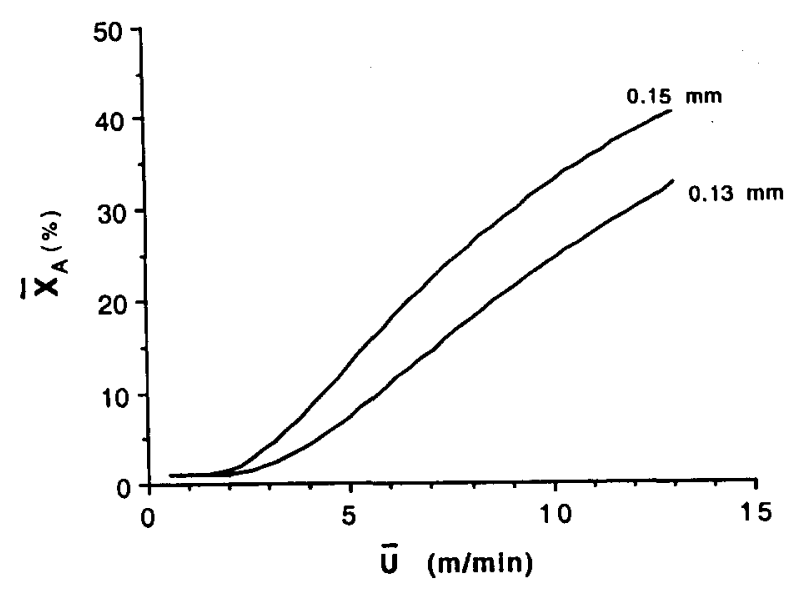

Fig. 9. Effect of impregnation velocity on volatile content for various film thickness.

data in Fig. 10. The experimental results were obtained by impregnating the reinforcements with different velocities, and following the procedures of ASTM D3530 for the determination of the volatile content. Theory agrees quite well with the experimental data. The discrepancy between is attributed to the assumption of an isothermal condition and the diffusion coefficient used in the computation. Equation (24) is plotted in Fig. 11. The results of film theory is superimposed for comparison. It is seen that the volatiles are most efficiently removed or transferred near the entrance region of a heating oven. This implies that an increase in the oven length beyond $D_{\mathrm{AB}} L /\left(\bar{U} \delta^{2}\right)=2$ is not useful in enhancing the mass transfer efficiency of the system.

An analysis is preformed to define the range of the validity in replacing $U_{y}$ with $\bar{U}$ in eqn (15). A substitution of $z^{*}=0$ into eqn (9) yields

$$
\begin{aligned}
\left(U_{\mathrm{y}}^{*}\right)_{z^{*}=0} & =1-\left(\frac{n}{n+1}\right)\left(\frac{\rho_{\mathrm{R}} g \delta^{n+1}}{\eta_{0} \tilde{U}^{n}}\right)^{1 / n} \\
& =1-\left(\frac{n}{n+1}\right)\left(N_{\mathrm{Im}}\right)^{1 / n}
\end{aligned}
$$

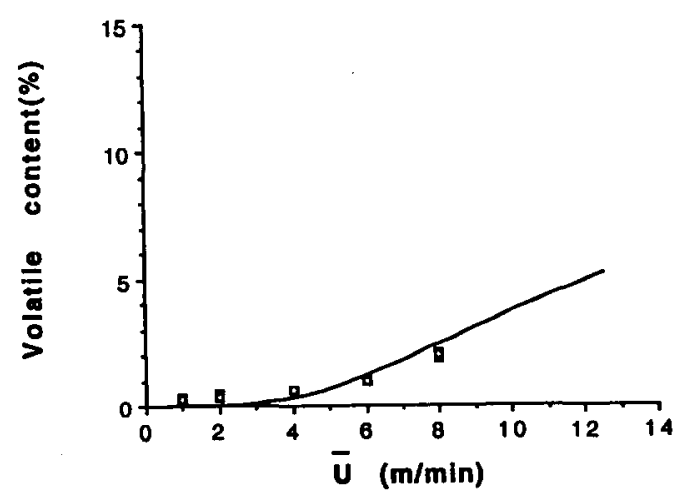

Fig. 10. Comparison between ( $\longrightarrow$ ) eqn (21) and ( $\square$ ) experimental results.

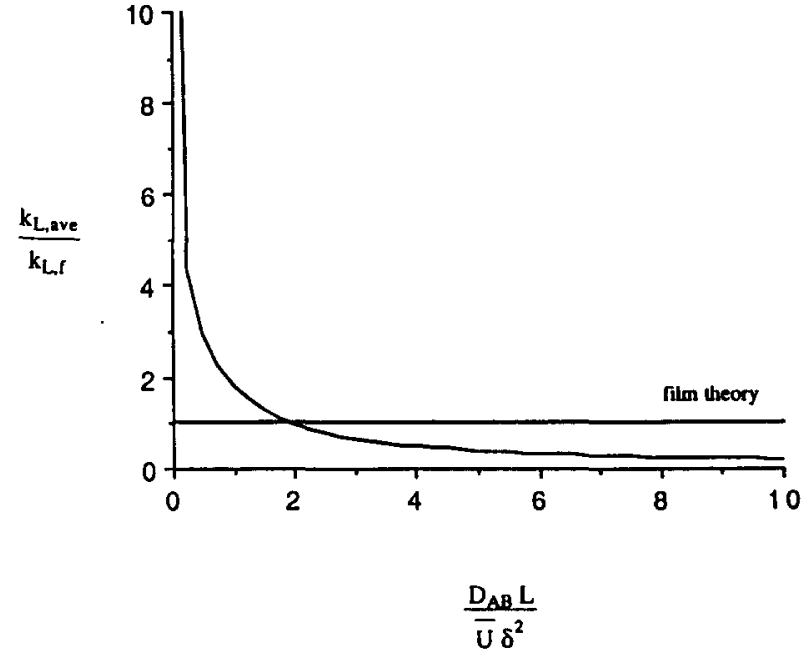

Fig. 11. Average mass transfer coefficient versus length of heating zone.

Figure 12 plots the dimensionless velocity, $U_{y}^{*}$, at $z^{*}=0$, versus $N_{\text {Im }}$ with the flow index as a parameter. For a shear-thinning fluid, the velocity at $z^{*}=0$ is almost unity for $N_{\text {Im }}$ less than 0.25 . It is therefore concluded that eqn (15) may approximate eqn (2) for shear-thinning materials in the range of $N_{\mathrm{Im}}$ less than $\mathbf{0} \cdot 25$. For a shear-thickening material, this approximation will only be valid for a very small value of $N_{\mathrm{Im}}$. Since an actual impregnation process of a shearthinning fluid, with a coated thickness of $0.1-0.3 \mathrm{~mm}$, has an apparent viscosity in the range of $75-500 \mathrm{cps}$ and an impregnation velocity of $3-8 \mathrm{~m} / \mathrm{min}$, the corresponding $N_{\mathrm{Im}}$ is about $10^{-2}-10^{-1}$. Therefore, eqn (21) is a valid approximation.

\section{CONCLUSION}

A mathematical model has been developed to describe the process of prepreg manufacture by

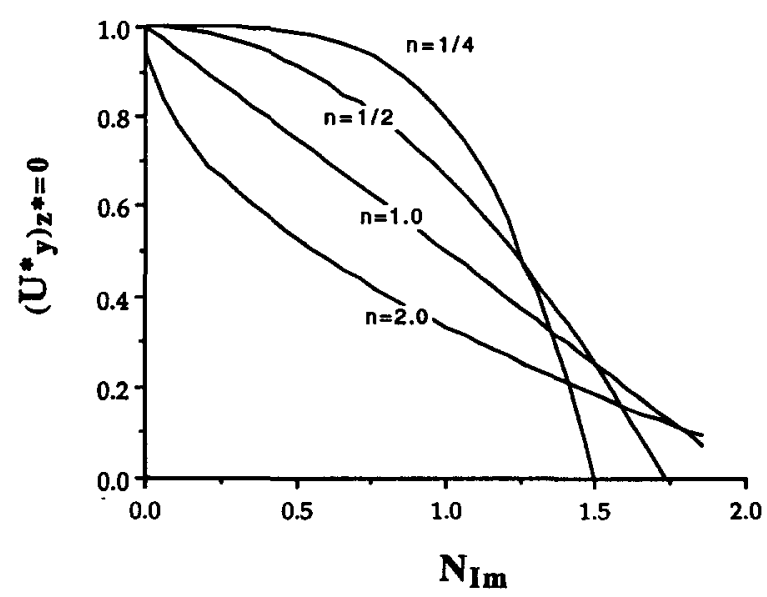

Fig. 12. Velocity on free surface versus Impregnation number. 
viscous coating. A dimensionless number, the impregnation number, has been derived which expresses the relative importance of the gravity, viscous and inertial forces in the process. It was found that increasing the impregnation velocity is less effective than increasing the viscosity in achieving a higher resin content of prepregs for impregnation with both Newtonian and shear-thinning resins. The influence of solid content on the final resin content is determined. The volatile content of prepregs is obtained as a function of the impregnation velocity and the heating length is also derived to give a possible combination of impregnation velocity and heating length to obtain a specific volatile content. Theoretical prediction of volatile content agrees well with experimental results. Results are found to be applicable to the practical impregnation process.

\section{REFERENCES}

1. Lindenmeyer, P. H. \& Sheppard, C. H., Characterization of PMR Polyimide Resin and Prepreg (NASA CR-168217). NASA, Cleveland, OH, 1984.

2. Keck, F. L., Formulation and Characterization of Epoxy Resin Copolymer for Graphite Composites (NASA CR-16650). NASA, Cleveland, OH, 1983.

3. Slobodzinsky, A., Bag molding process. In Handbook of Composite Materials, ed. G. Lubin. Van Nostrand
Reinhold Company, New York, USA, 1982, pp. 368-90.

4. Sanjana, S. N., Overage indication for prepreg products. SAMPE J., 16 (1980) 5-11.

5. Day, D. \& Shepard, D., Effect of advancement on epoxy prepreg processing-A dielectric analysis. Polym. Comp., 12 (1991) 87-90.

6. Cole, K. C., Noel, D., Hechler, J.-J., Chouliots, A. \& Overbury, K. C., Room temperature aging of Narmo 5208 carbon-epoxy prepreg. Part I. Polym. Comp., 10 (1989) 150-61.

7. Yue, C. Y., Differential scanning calorimetric determination of the volatile content and properties of epoxy prepreg. J. Mat. Sci. Lett., 7 (1988) 811-13.

8. Deryagin, D. V., Theory of the deposition of a viscous liquid onto a fiber or wire being withdrawn from the liquid. Prinkl. Mekh. Teckh. Phys., 3 (1963) 71-8.

9. Levy, H. L. R. M., Lockyer, A. J. \& Arridge, R. G. C., The coating of fiber. Int. J. H. Mass Transfer, 21 (1978) 435-43.

10. Rezaian, A. \& Poulikakos, D., Heat and fluid flow processes during the coating of a moving surface. $J$. Thermophysics, 15 (1991) 192-8.

11. Cheung, F. B., Analysis of freeze coating on a nonisothermal moving plate by a perturbation method. J. Heat Transfer, 107 (1985) 549-56.

12. Sherwood, T. K., Pigford, R. L. \& Wilke, C. R., Mass Transfer. McGraw-Hill, New York, USA, 1975, pp. 25-7.

13. Cussler, E. L., Diffusion, Mass Transfer in Fluid System. Cambridge University Press, Cambridge, UK, 1984 , chapter 5 